\title{
American Canine Hepatozoonosis
}

\author{
S. A. Ewing* and R. J. Panciera \\ Department of Pathobiology, College of Veterinary Medicine, Oklahoma State University, \\ Stillwater, Oklahoma 74078
}

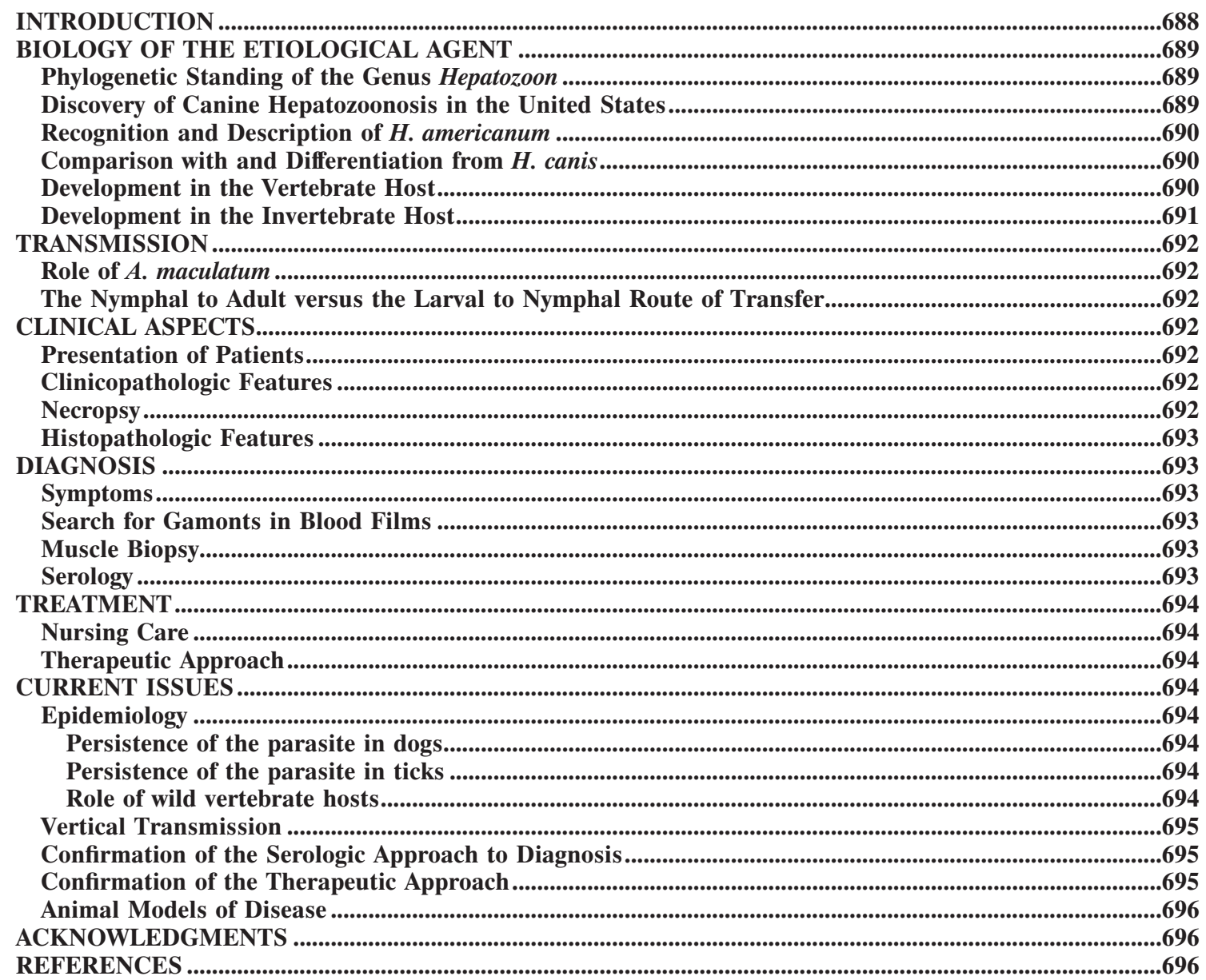

\section{INTRODUCTION}

American canine hepatozoonosis $(\mathrm{ACH})$ is an emerging disease of dogs (Canis familiaris) in the south-central and southeastern United States. It is a highly debilitating, tick-borne malady that is spread not by the bite of ticks but by dogs ingesting infected ticks. When first discovered in the Gulf Coast region in the late 1970s, it was mistakenly thought to be an Old World canine disorder caused by Hepatozoon canis. In the 1990s, veterinarians and veterinary medical researchers came to realize that the New World malady was decidedly more pathogenic than classical canine hepatozoonosis caused

\footnotetext{
* Corresponding author. Mailing address: 250 McElroy Hall, Oklahoma State University College of Veterinary Medicine, Stillwater, OK 74078. Phone: (405) 744-8177. Fax: (405) 744-6633. E-mail: saewing @okstate.edu.
}

by $H$. canis $(39,53,54,55,63)$. This realization, coupled with other incongruities between the two disorders, led to studies that resulted in the description of a new species, $\mathrm{H}$. americanum (62). The species was recognized in 1997, and the disease and its transmission pattern have been characterized, including discovery that the Gulf Coast tick, Amblyomma maculatum, is an excellent vector $(21,41)$. It has been posited that $H$. americanum crossed the species barrier into canids from unknown vertebrates only recently whereas $H$. canis apparently has a long history of association with dogs (4). The endemic cycle of $H$. americanum remains unknown, and it is unclear where ticks acquire infection or under what circumstances dogs are exposed to infected ticks. We speculate that they become infected either through grooming infected ticks from their coats or from ingesting such ticks accidentally as they maul or eat prey that harbor the ticks. 


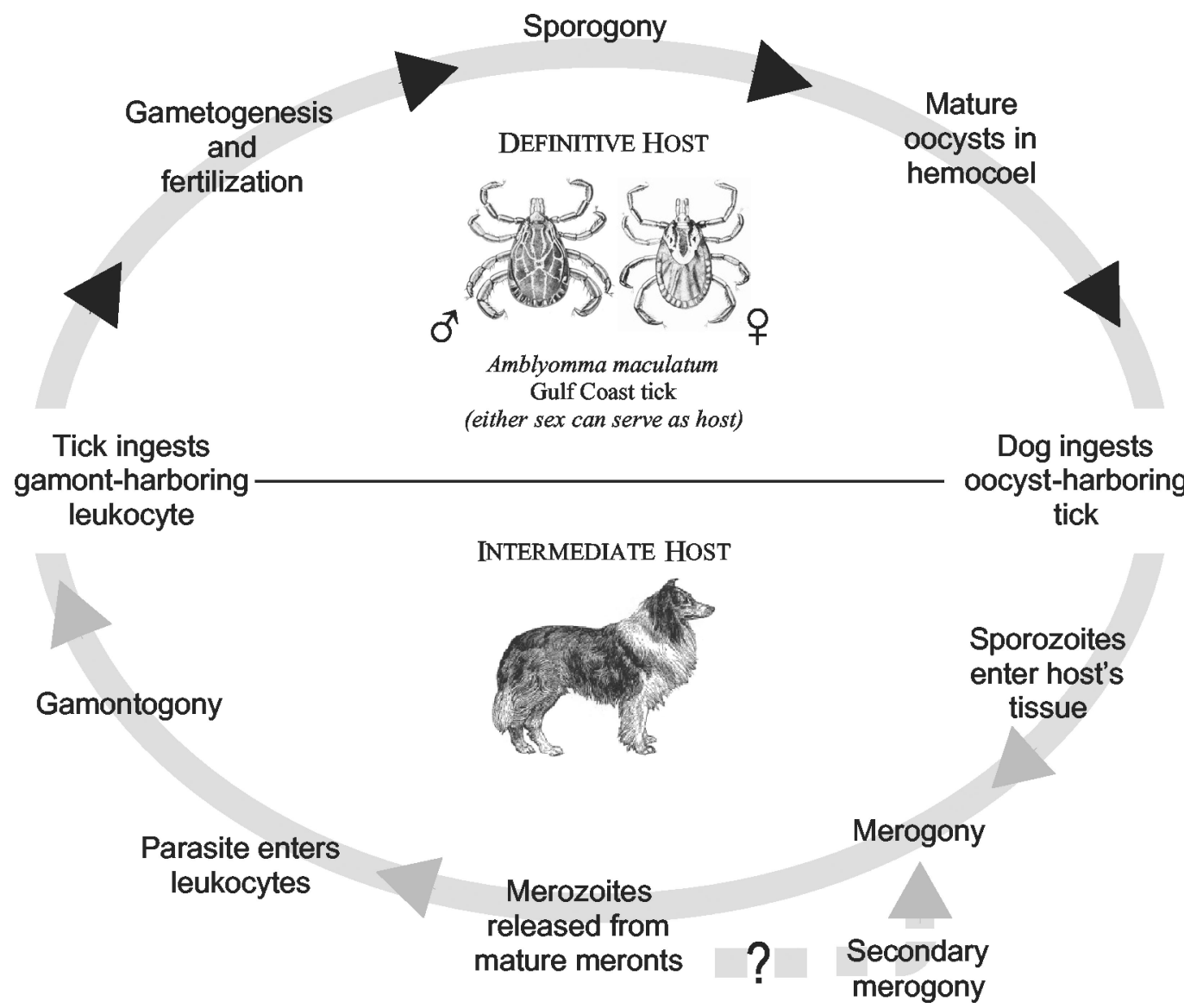

FIG. 1. Life cycle of H. americanum. Gulf Coast ticks, the definitive host for H. americanum, ingest gamont-containing leukocytes. Sexual reproduction occurs within the tick, followed by zygotic meiosis and sporogony, an asexual multiplicative process. Vertebrate intermediate hosts, including dogs, ingest ticks that harbor oocysts that rupture, releasing sporocysts that in turn excyst in the presence of bile, releasing infectious sporozoites. Sporozoites invade host cells and give rise to a second asexual multiplicative process, merogony. When meronts mature, they rupture, releasing merozoites, which give rise to gamonts that circulate in the blood, from where ticks may ingest them. Secondary merogony is thought to occur (see the text).

\section{BIOLOGY OF THE ETIOLOGICAL AGENT}

\section{Phylogenetic Standing of the Genus Hepatozoon}

The Hepatozoon species belong to the phylum Apicomplexa and have many features in common with other genera in this large group of parasitic protozoans. Formerly a part of the family Haemogregarinidae, Hepatozoon is a large genus comprising about 300 species that is now assigned to the family Hepatozoidae of the suborder Adeleorina $(6,7,59)$. At least 46 species are found in mammals, and more than 120 are found in snakes. These apicomplexan parasites are spread to vertebrate intermediate hosts by hematophagous invertebrates that are definitive hosts and simultaneously also vectors. Like relatives such as Plasmodium spp. and Babesia spp., which inhabit erythrocytes in the bloodstream of vertebrates, many Hepatozoon spp. (especially of snakes) are found in erythrocytes (59). Most species that parasitize mammals, including $H$. americanum and H. canis, inhabit leukocytes and utilize acarines as definitive hosts or vectors. Exceptions include Hepatozoon balfouri, a highly pathogenic parasite of jerboas (rodents of the genus Jaculus), which is found in erythrocytes; the definitive hosts of this species are laelapid mites (25). Unlike numerous parasites that are spread by the bite of arthropods, Hepatozoon spp. are acquired when the vertebrate host ingests the infected invertebrate (Fig. 1).

\section{Discovery of Canine Hepatozoonosis in the United States}

The first reported naturally occurring Hepatozoon infection of canids in the New World was in 1978, when a single infected coyote (Canis latrans) was found in the Aransas National Wildlife Refuge near Aransas Pass, Tex. (17). The authors speculated that it was likely that $H$. procyonis, a parasite previously known to be in that part of Texas, had crossed from the raccoon (Procyonis lotor) to the coyote (12). Shortly thereafter, others reported finding dogs (C. familiaris) ill with hepatozoonosis in the Texas Gulf Coast region (13). Parasites that appeared identical to those described in dogs in India $(9,10,30$, 64 ) in the early 1900s were found in peripheral blood leukocytes, and it was concluded that $H$. canis had entered the United States (13). Workers at Texas A \& M University characterized the disease in dogs and pointed out certain differences with the Old World disease. These workers had limited success in attempts to transmit the disease experimentally, and 
thus their studies were done largely with naturally infected dogs (51). Based on knowledge of the disease in dogs in Africa, Asia, and southern Europe, where Rhipicephalus sanguineus was known to serve as the vector, they were puzzled by their limited success with transmission studies that involved this tick. Among nine dogs exposed to ticks that fed on a naturally infected dog, only two developed disease, and these were the youngest animals exposed. Moreover, only one oocyst was found in 120 experimentally exposed brown dog ticks dissected (51). We now know that $R$. sanguineus is refractory, at least to the $H$. americanum isolates studied in Oklahoma $(21,23)$.

\section{Recognition and Description of $\boldsymbol{H}$. americanum}

Workers at Auburn University reported severely debilitating hepatozoonosis in dogs from Alabama and western Georgia (39). After diagnosing a large number of cases and recognizing that the parasite was more pathogenic than the Old World form, additional studies led the authors to propose a new species, which they assigned the specific epithet "americanum," a decision that has been endorsed by others (3, 29, 43, 62). Among others, a major difference between $H$. canis and $H$. americanum that justified the definition of a new species included failure to infect $R$. sanguineus with the organism.

\section{Comparison with and Differentiation from $H$. canis}

$H$. americanum gamonts are found in circulating leukocytes of dogs, as are those of $H$. canis. Electron microscopy and immunohistochemical evidence indicates that the host cell for $H$. americanum during merogony and gamogony is a monocyte, rather than a neutrophil, which is considered the favored host cell for $H$. canis (16). Also, merogony of $H$. americanum takes place in a host cell that is lodged primarily between individual striated muscle fibers whereas this asexual process for $H$. canis occurs in a wide variety of sites, especially in hemolymphatic tissues and visceral organs. The meronts of $H$. americanum are usually found within "onion skin" cysts that are created by layers of mucopolysaccharide-rich material that is apparently elaborated by the host cell $(16,19,54)$. No such characteristic lesion is associated with the $H$. canis meront, which is rarely found in muscle and has its own characteristic morphologic feature referred to as a "wheel spoke" arrangement of merozoites within the meront (4).

\section{Development in the Vertebrate Host}

When dogs ingest ticks that contain mature H. americanum oocysts, these fragile-walled structures are believed to quickly rupture, releasing the stout-walled sporocysts. Each sporocyst is packed with sporozoites, the infective stage for the vertebrate intermediate host. When sporocysts are exposed to dog bile experimentally, the sporozoites are released within minutes, and it is assumed, therefore, that the infective stages are released in the canine gut distal to where the common bile duct empties; however, the process has not been observed experimentally in the canine gut. It is assumed, also, that the sporozoites cross the gut wall and are carried via lymphatics or the bloodstream to tissues throughout the body; it is not known whether the parasite is transported extracellularly or within the host cell in which merogony eventually occurs. A parasitized host cell has been demonstrated to be lodged between myofibers in a variety of skeletal muscles soon after experimental exposure to infective oocysts $(16,55)$. In any case, the trophozoite that has been identified within macrophage-like cells in many tissues, principally striated muscle, apparently transforms the host cell into a mucopolysaccharide-producing entity that builds the structures commonly called onion skin cysts (Fig. 2A).

The developing $H$. americanum organism apparently is shielded from the dog's immune system until merogony is completed and the cystic structure is breached. The mature meronts release merozoites, which incite intense local inflammation, associated with a systemic reaction and overt illness. The local lesions evolve to highly vascular granulomas; parasites are present in the macrophages of granulomas, where, presumably, gamogony commences (54). Parasites enter leukocytes, which subsequently circulate in the bloodstream as gamonts and may be consumed by ticks taking a blood meal. It has been postulated that certain merozoites do not become gamonts but, rather, give rise to a new merogonous cycle; however, the phenomenon has not been demonstrated experimentally. Meronts that produce micromerozoites and others that produce macromerozoites have been described, and the fate of these stages may differ $(13,28)$. With $H$. domerguei, a cystozoite stage is recognized that is capable of surviving indefinitely in vertebrate hosts; this stage retains the ability to give rise to meronts like those that are derived from sporozoites and develop when the cystozoite-harboring host is ingested by a susceptible vertebrate host (36). Similarly, $H$. griseisciuri has small cystic stages in the lungs of squirrels that may give rise to infection in hosts that eat them (18).

Indirect evidence that some $H$. americanum merozoites give rise to new merogonous generations rather than to gamonts is provided by observations of a naturally infected dog that remained infected for at least 5.5 years. Maintained without parasiticidal therapy or opportunity for reexposure, this dog had demonstrable muscle cysts and its blood was still infectious for Gulf Coast ticks when testing was discontinued (24). The possibility cannot be discounted, however, that sporozoites taken in initially by this dog had simply lain quiescent until giving rise to meronts months to years later, similar to the phenomenon of delayed development seen in relapses of some Plasmodium infections that involve the development of hypnozoites (33). Sporozoites of $H$. domerguei are known to have two distinctive routes of development, and we cannot dismiss that possibility for $H$. americanum. The theory of sporozoite polymorphorism postulated for Plasmodium vivax (the causative agent of tertian malaria of humans) might also account for the phenomenon observed with $H$. americanum (38).

There is also some direct evidence that $H$. americanum has two kinds of parasites in granulomas and in peripheral blood leukocytes. It is suspected but not known that these structures represent both gamonts and merozoites (16). It is also possible that some of these structures are comparable to the polymorphic forms described for $H$. domerguei or for various Plasmodium spp. $(33,36)$. 

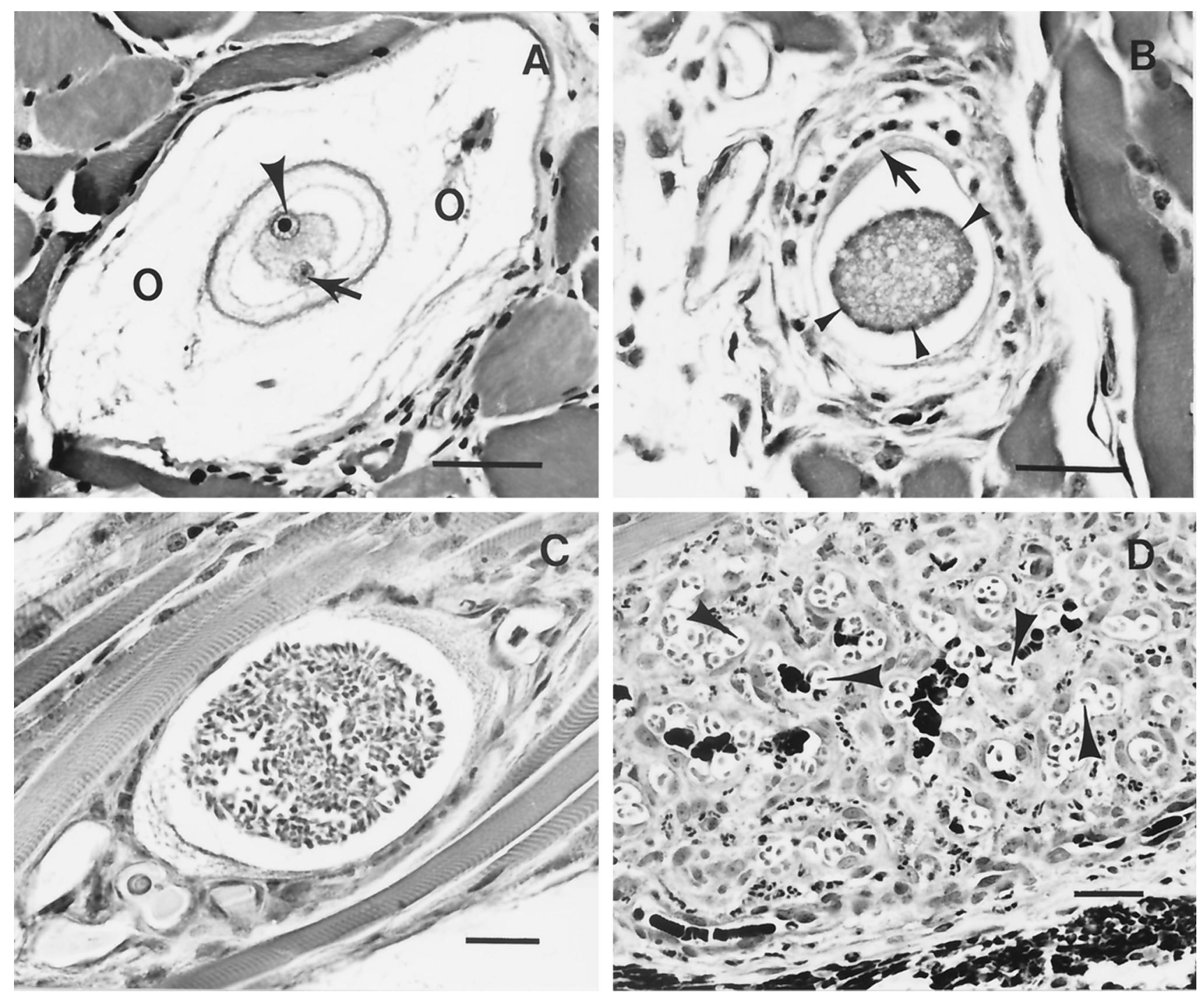

FIG. 2. Light photomicrographs of stages of $H$. americanum developing in canine skeletal muscle. (A) Onion skin cyst (O) containing a host cell with a nucleus (arrowhead) and its large nucleolus. Notice the intracytoplasmic zoite (arrow). Cyst material is composed of irregularly lamellated acid mucopolysaccharide produced by the infected host cell. (B) Early-stage meront with nuclear material aggregating at the margins (arrowheads). Notice the host cell cytoplasm (arrow). (C) Meront containing well-differentiated merozoites. (D) Pyogranuloma in which macrophages harbor intracytoplasmic parasites (arrowheads). Bars, $50 \mu \mathrm{m}$ (A and D) and $25 \mu \mathrm{m}$ (B and C).

\section{Development in the Invertebrate Host}

Most of the work on the development of $H$. americanum in its ixodid definitive host was done with ticks that were infected experimentally as nymphs and then studied during digestion of the blood meal and during the molting process as they transformed into adults (42). The only tick species in which development has been studied is $A$. maculatum, a tropical species commonly called the Gulf Coast tick. Nymphs that fed experimentally on dogs with either natural or experimentally induced $\mathrm{ACH}$ readily became infected. The gamonts presumably are released from the canine leukocytes soon after the tick ingests them, but the male and female gamonts have not been differentiated microscopically in the tick gut lumen. However, structures that appear to be microgametes and macrogametes, seen about 6 days after the ticks began their blood meal, have been observed in tissue sections of newly fed nymphs. Shortly after that, at about 9 days, zygotes were observed within cells of the tick's gut wall. Like Plasmodium spp. in mosquitoes, $H$. americanum appears to be diploid only briefly. What appeared to be reduction division was observed at about 20 days after exposure or approximately 2 weeks after the nymphs, replete with blood, left the dog. Once the parasite returns to the haploid state by zygotic meiosis, sporogony is completed within the tick gut cells. This asexual reproductive process gives rise eventually to oocysts packed with masses (hundreds) of sporocysts, each containing 10 to 26 sporozoites (42). As oocysts develop, they distort the host cell, which bulges into the tick's hemocoel. Some of these oocysts become dislodged and are free in the body cavity (hemocoel); they are mechanically released when the tick's body is ruptured on ingestion by dogs or other vertebrate intermediate hosts. 


\section{TRANSMISSION}

\section{Role of $A$. maculatum}

H. americanum, like other species in the genus, is apparently more restricted in host range in hematophagous invertebrate definitive hosts than in vertebrate intermediate hosts. Recent work indicates that $A$. maculatum is an excellent host whereas three other more common ixodids (namely, $R$. sanguineus, Dermacentor variabilis, and $A$. americanum) that were exposed by experimental feeding are refractory to infection (23). It appears, therefore that, in the United States at least, only the Gulf Coast tick is commonly a vector. (Ixodes scapularis, the only other likely candidate vector in areas where ACH is endemic, has not been tested.) Recent reports from South America suggest that $A$. cajennense should also be tested; dogs in Brazil were more apt to have hepatozoonosis when this tick was present than when $R$. sanguineus or other ticks were found (52). Although it was unclear whether the parasite causing disease in these largely rural Brazilian dogs was $H$. canis, $H$. americanum, or another undescribed Hepatozoon spp., the observation that parasitemia was minimal suggests that the agent was not $H$. canis, which routinely produces high parasitemia. A. cajennense is also a tropical tick species sometimes reported in the southern United States; it has not spread as widely in North America as has $A$. maculatum. The latter was once thought not to be endemic beyond a range of about 200 miles from the Gulf Coast, whereas it now is endemic quite far inland, including northeastern Oklahoma and southeastern Kansas $(58,60)$. In Brazil, A. cajennense ticks found on dogs were usually larvae or nymphs; favored hosts of the adults were equids (52).

Japanese workers (50) have found oocysts in Haemaphysalis spp. taken from dogs with hepatozoonosis. It is unclear whether the oocysts are those of H. canis or some other Hepatozoon spp.

The apparently restricted definitive host and vector range suggests that $H$. americanum, unlike disease agents transmitted to dogs by $R$. sanguineus, will not spread into colder or drier parts of the United States. In contrast, for example, canine ehrlichiosis, caused by Ehrlichia canis, is found in all parts of the United States, wherever the brown dog tick is found. Although originally from the Old World, the brown dog tick was introduced around 1910 and is widespread in North America, where it survives readily in houses and kennels and feeds in all stages almost exclusively on dogs (34). In contrast, the Gulf Coast tick feeds on a variety of different hosts in larval, nymphal and adult stages and is very susceptible to desiccation; it requires high relative humidity to survive. Under natural conditions it must also have access to an array of hosts in order to complete its life cycle $(58,60)$. Most dogs with naturally occurring $\mathrm{ACH}$ are of rural origin and thought to have acquired infection in such a setting. This observation is another indication that $R$. sanguineus is not involved in the natural history of $H$. americanum.

\section{The Nymphal to Adult versus the Larval to Nymphal Route of Transfer}

Larval Gulf Coast ticks can acquire infection with $H$. americanum, and nymphs developing from replete larvae that fed on a dog with $\mathrm{ACH}$ have been shown to contain infective oocysts (22). These recent observations indicate that the task of elucidating the natural cycle of this disease agent will be a difficult one because the host range of larval $A$. maculatum, although not well studied, is known to include a variety of birds and mammals $(5,58,60)$. Accordingly, investigation of the cycle of endemicity is complicated and discovery will be delayed until thorough studies of candidate wildlife species can be completed. More extensive information than is currently available on the feeding range of larval and nymphal Gulf Coast ticks would facilitate such investigations. Canids that eat rodents or other small vertebrates on which the ticks feed would easily bite, rupture, and ingest infected nymphal ticks. There are no data available to suggest whether dogs acquire $\mathrm{ACH}$ primarily by eating ticks while grooming or by ingesting nymphal or adult ticks as a consequence of eating or mauling animals infested with infected Gulf Coast ticks.

\section{CLINICAL ASPECTS}

\section{Presentation of Patients}

Diseased dogs are often febrile, stiff, lethargic, and depressed. Gait abnormalities and muscle wasting are usually obvious, as is copious mucopurulent ocular discharge. Atrophy of head muscles is especially noticeable. Dogs may eat readily when food is placed immediately in front of them, but they often refuse to move to food and water, presumably owing to intense pain, which derives in part from periosteal bone proliferation and inflamed muscles $(15,39,53,63)$.

\section{Clinicopathologic Features}

Persistent, generally mature neutrophilia is a consistent feature of naturally occurring $\mathrm{ACH}$ (26). Leukocyte counts greater than $200,000 / \mu l$ may be seen. Serum alkaline phosphatase levels increase with concurrent decrease in serum protein and albumin levels $(39,63)$. These clinicopathologic features were also observed in experimentally induced disease (16). Artifactual hypoglycemia is sometimes observed if samples are not processed quickly, a feature resulting from metabolism of glucose by the numerous leukocytes found consistently in dogs with $\mathrm{ACH}(39,63)$.

Radiographs reveal extensive periosteal bone proliferation. Lesions occur more frequently and more severely on the proximal bones of the limbs. Distal limb bones are usually spared $(20,56)$. Flat bones are less frequently but sometimes markedly affected. In experimentally induced disease, bone scintigraphy and radiography reveal lesions as early as 5 weeks after dogs ingest infected ticks or oocysts dissected from experimentally infected ticks (16). Similar periosteal lesions have been found in Japanese dogs with $H$. canis infection (48).

\section{Necropsy}

Gross lesions are generally limited. Wasting of body mass, especially marked in temporal muscles, is often seen in dogs with longstanding disease. Moderate enlargement of body lymph nodes and spleen is common. Periosteal bone proliferation is obvious in most dogs, including those experimentally exposed as recently as 6 weeks previously (16). Limb edema 
may be present in dogs that have been recumbent. Occasionally dogs have peritoneal effusion associated with myocarditis that results from merogonous stages in the heart or from protein loss owing to glomerulopathy $(53,63)$.

\section{Histopathologic Features}

Parasite-containing lesions can be found in many tissues, but they are consistently found in striated muscle (including cardiac muscle) where the parasite undergoes merogony (Fig. 2A to C). Marked pyogranulomatous myositis occurs following liberation of merozoites from the intracellular environment (Fig. 2D). Adipose tissues, as well as loose connective tissues, are less commonly affected; rarely, other organs and tissues such as the lymph nodes, spleen, liver, and pancreas have lesions $(54,63)$. In contrast, $H$. canis merogonous stages are found most often in the spleen and other hemolymphatic locations, not in muscles (4).

Periosteal lesions of $\mathrm{ACH}$ are similar to those of hypertrophic osteopathy in dogs and other animals; they are essentially identical to those of hypertrophic osteoarthropathy of humans (56). There are no parasites intimately associated with the bone lesions. Although the inciting factor(s) of these osteal lesions found in dogs with $\mathrm{ACH}$ is not known, it is assumed that cytokines elaborated by the animal in response to the infection are involved; however, experimental evidence to support this assumption is lacking (16).

\section{DIAGNOSIS}

\section{Symptoms}

Dogs suffering from $\mathrm{ACH}$ are periodically or persistently febrile; this is probably associated with a host reaction to merozoites that are released when meronts rupture. Weakness and muscle atrophy is obvious in most dogs, and generalized pain, presumably resulting from myositis and from periosteal bone proliferation, is common. Some dogs are reluctant to rise even to seek food but will continue to eat and drink while terminally ill. Extensive bilateral mucopurulent ocular discharge is common during febrile episodes $(8,13,39,53)$.

\section{Search for Gamonts in Blood Films}

Diagnosis of ACH by examination of blood films stained with Romanowsky-type stains is problematic because leukocytes containing H. americanum gamonts (Fig. 3) can be found only rarely, probably with much less than $0.1 \%$ of circulating white blood cells being affected $(27,63)$. Even with special staining efforts, searching blood films is frustrating (46). Consequently, irrespective of sample preparation, laborious searching of blood films is largely nonproductive as a means of diagnosing ACH. This is decidedly different from confirmatory diagnosis of $H$. canis infection. Because parasitemia is usually marked in $H$. canis infection, diagnosis of that infection is readily confirmed by examination of blood smears; with some exceptions (35) when low levels have been reported, rates of parasitemia in $H$. canis infections are commonly 1 to $5 \%$, and up to $70 \%$ parasitemia is sometimes seen in the numerous peripheral blood neutrophils that usually circulate in dogs with the disease $(1,2)$. ACH can confidently be predicted in dogs that

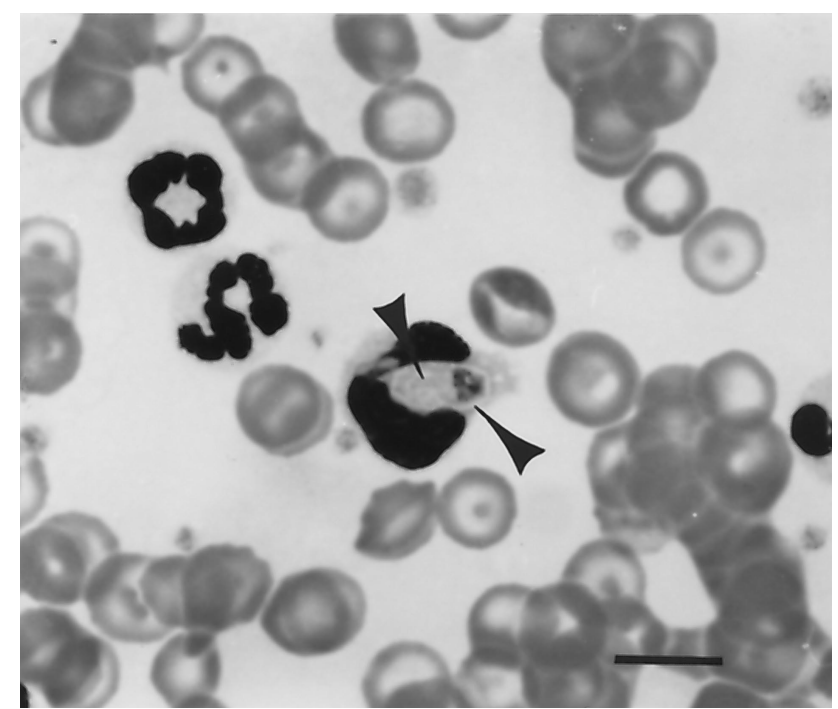

FIG. 3. H. americanum gamont (arrowheads) within a peripheral blood leukocyte. Bar, $10 \mu \mathrm{m}$.

have profound neutrophilia and periosteal bone lesions concurrently, even in the absence of demonstrable parasites in leukocytes.

\section{Muscle Biopsy}

Confirmatory diagnosis of $\mathrm{ACH}$ is achieved by biopsy of skeletal muscle $(8,14,55)$. Often there are surprisingly numerous merogonous stages of $H$. americanum in a wide range of muscles. (No such cysts are found in dogs with $H$. canis infections.) Site selection for biopsy is largely a matter of personal preference and is related to surgical procedure rather than to the abundance of parasites in any given muscle (55). Although routine hematoxylin-eosin stains are adequate, a highly effective immunohistochemical procedure utilizing rabbit antibody to sporozoites of $H$. americanum as the primary reagent is available (57).

\section{Serology}

Although not widely available to date, an indirect enzymelinked immunosorbent assay has been developed (44). The antigen for the test involves $H$. americanum sporozoites derived from experimentally infected ticks. The fragile oocysts are ruptured mechanically to release sporocysts. These stoutwalled sporocysts are induced to excyst ("hatch") by exposure to dog bile. Large numbers of sporocysts are easily recoverable, but to date the sporozoite preparations have not been successfully cleared of residual tick tissues that adhere to oocyst walls and thus contaminate the antigen. This contamination necessitates the adsorption of any sera to be tested because tick exposure is so common among dogs in areas where $\mathrm{ACH}$ is endemic. Although other reagents would probably be satisfactory for adsorption, those used to date were from tick gut tissue collected from uninfected laboratory-reared $A$. maculatum (44). 


\section{TREATMENT}

\section{Nursing Care}

Dogs with ACH may experience intense pain and become reluctant to move. Therefore, efforts must be made to ensure that affected animals remain hydrated and that food is readily accessible. Supportive care is important. Pain control has been achieved with nonsteroidal anti-inflammatory drugs $(15,63)$. It is likely that some dogs with $\mathrm{ACH}$ that is never diagnosed recover as a result of good care by owners.

\section{Therapeutic Approach}

A treatment protocol has been developed that is effective in alleviating overt disease. It involves a combination of trimethoprim-sulfadiazine, clindamycin, and pyrimethamine administered daily for 14 days. This regimen is followed by decoquinate, an effective anticoccidial drug, for years. Unfortunately, if the protocol is not strictly adhered to, relapse is likely to occur within weeks to months after decoquinate treatment is discontinued (40). Decoquinate is a quinolone anticoccidial that is effective against intracellular stages of certain apicomplexan parasites, and relapses are known to occur if treatment with the drug is suspended (37). For example, chickens that harbor inhibited coccidian sporozoites will develop coccidiosis when the drug is no longer administered. Presumably, a similar event occurs with $H$. americnum infections, but the phenomenon has not been studied to determine which stage(s) in the life cycle is affected. Imidocarb is used extensively for treating $H$. canis infections, but its effectiveness for treating $\mathrm{ACH}$ has been questioned (40).

\section{CURRENT ISSUES}

\section{Epidemiology}

The natural cycle of $H$. americanum is not known. It is known, however, that dogs acquire infection by ingesting either nymphal or adult $A$. maculatum ticks that harbor sporozoitecontaining oocysts $(21,22,41)$. Determining how the ticks become infected under natural conditions is an important undertaking. These ixodids are easily infected under experimental conditions by simply allowing larval or nymphal ticks to feed on a carrier dog. A. maculatum is a three-host tick and, accordingly, must feed to repletion as a larva, as a nymph, and as an adult (58). Determining the source of infection for ticks under natural conditions will require study of the natural hosts of the ticks. Dogs are not among the favored hosts, although larvae, nymphs, and adults all feed to repletion on this host under experimental conditions. The problem of discovering the endemic cycle of $H$. americanum is complicated by the somewhat incomplete state of knowledge of host preferences of the Gulf Coast tick, especially the larval stage.

Persistence of the parasite in dogs. In the absence of knowledge of the natural cycle, we are presently restricted to drawing inferences from the behavior of $H$. americanum in the dog. A naturally infected dog kept under conditions that excluded reexposure was monitored for 5.5 years. This dog had been moribund when first diagnosed but was successfully nursed back to health with pain control and supportive therapy con- sisting of little more than good nutrition and comfortable housing. Aspirin was the only drug used. Gulf Coast ticks were allowed to feed at approximately 6-month intervals; each time they acquired infection (24). Significantly perhaps, the dog was asymptomatic for $\mathrm{ACH}$ for the last 5 years of observation. Throughout much of the time, however, the dog had mild neutrophilia, and merogonous stages of $H$. americanum were demonstrable by muscle biopsy in 13 of 15 specimens over the 5.5-year span (24).

Coyotes are known to harbor a parasite that is similar if not identical to $H$. americanum, but information is not available about the persistence of the organism in that canid. Given that approximately $50 \%$ of "healthy" coyotes captured in Oklahoma have lesions nearly identical to those of $\mathrm{ACH}$, we infer that infections persist in this host for extended periods. Coyotes have been infected experimentally with $H$. americanum by feeding them with oocysts derived from ticks that fed to repletion on dogs harboring the parasite $(31,32)$. Although naturally infected coyotes are numerous in Oklahoma, no attempt has been made to assess the survival time of $H$. americanum in these animals.

Persistence of the parasite in ticks. Little work has been done to determine longevity of Gulf Coast ticks experimentally infected with $H$. americanum, but we have held infected adult ticks in humidity chambers for a year or more following exposure as nymphs. We have not conducted experiments to determine whether the infection status affects longevity, but anecdotal evidence suggests that heavily infected ticks start to die before one would expect, based on survival of unfed adult $A$. maculatum ticks reared in the laboratory (unpublished data).

There are no reports of observations of naturally infected ticks recovered in the wild. In the southern United States, where $\mathrm{ACH}$ has been studied, $A$. maculatum produces one generation per year. In this temperate zone, larval stages feed mainly in summer and nymphs feed principally in autumn. Overwintering appears to be done mostly by unfed adults, although nymphs have been found feeding in Oklahoma in late winter, indicating that unfed nymphs can also overwinter $(5,58)$.

Irrespective of the tick biology, in view of the extended survival of $H$. americanum in the vertebrate host, it seems unlikely that long-term survival in its definitive host or vector is a major factor in maintaining the parasite in its region of endemicity. It is not known whether larval or nymphal ticks are more important in acquiring infection in the natural cycle, nor is it known which tick stage is more often a source of infection for dogs. Experimentally, both larval and nymphal stages consistently acquire infection even though very few gamont-infected circulating leukocytes are demonstrable when the ticks are feeding $(21,22,41,42)$. Careful study is required to determine the endemic host range, and only then will the source of infection for ticks, later ingested by dogs, become clear.

Role of wild vertebrate hosts. In the 1970s, Hepatozoon spp. were studied in a wide range of wild carnivores in Kruger National Park in South Africa (45), where it was found to be most pathogenic in jackals (Canis mesomelas) and apparently relatively harmless in hyenas (Hyaena crocuta) and large felids (lions, leopards, and cheetahs). It appeared that the parasite in these wild carnivores was most probably $H$. canis, with the only demonstrable difference being slight variations in the meronts found in these wild species compared with those found in the 
dog. Some 20 years later, others studying the decline of the wild-dog (Lycaon pictus) population in Kruger National Park found that Hepatozoon (thought to be $H$. canis) infection was very prevalent, but its role in the decline of the wild dogs was unclear (61). Although no similar systematic study of carnivores has been undertaken in areas where $\mathrm{ACH}$ is endemic, a survey of carnivores in south Texas (47) revealed Hepatozoon spp. in coyotes, bobcats (Lynx rufus), and ocelots (Felis pardalis), all without evidence of disease (47).

It is tempting to speculate that coyotes are the major or only important vertebrate host of $H$. americanum. Coyotes harbor a parasite $(17,31)$ that is similar, if not identical, to $H$. americanum, and this wild canid has been successfully infected experimentally with $H$. americanum of dog origin (32). Furthermore, coyotes have been found to harbor immature as well as adult A. maculatum ticks under natural conditions $(5,58)$. Nevertheless, these facts alone do not justify the conclusion that coyotes and Gulf Coast ticks constitute the definitive and intermediate endemic hosts for $H$. americanum. Coyotes, like dogs, groom ticks from their coats and could become infected by ingesting infected ticks or simply the oocysts themselves that might be released when the ticks are damaged by the animal's teeth. Grooming behavior is not necessarily the most common or efficient means of acquiring infection, however. Canids are predatory and eat a wide variety of small vertebrates, including both birds and mammals. Such prey animals no doubt often carry Gulf Coast ticks, some of which may be infected with $H$. americanum. Determining which vertebrate host(s) is responsible for infecting the ticks is an important challenge.

Two hosts, raccoons and squirrels, which are very prevalent in areas where $\mathrm{ACH}$ is endemic, are known to harbor $H$. procyonis and $H$. grieisciuri, respectively. It should be relatively easy to determine through molecular techniques whether these species are closely related or identical to $H$. americanum. At present, it is assumed that all three species are valid, each having a distinct endemic cycle, but the matter has not been explored experimentally. The suggestion that $H$. americanum recently passed the species barrier (4) to enter dogs could be given substance by comparing Hepatozoon isolates from raccoons, squirrels, coyotes, and rodents with the parasite recovered from dogs with naturally occurring $\mathrm{ACH}$.

We have only a scant basis for speculating about the endemic cycle of $H$. americanum. If opportunity were available to undertake such an investigation, we would suggest looking at rodents as the primary vertebrate intermediate host that alternates with the Gulf Coast tick definitive host in maintaining $H$. americanum in the natural world. This opinion derives in part from knowledge that rodents are especially favored hosts (59) for Hepatozoon spp. More concretely, the information known about $A$. maculatum host preferences also suggests that rodents, especially cotton rats (Sigmodon hispidus), should be studied as possible hosts for $H$. americanum. A recent study (5) revealed that about $35 \%$ of cotton rats (134 of 384) captured in an area where $\mathrm{ACH}$ is endemic had larval Gulf Coast ticks on them and almost 19\% (71 of 384) harbored nymphs. Given that canids commonly eat cotton rats, this rodent might profitably be studied as a potential host for $H$. americanum and source of ticks infected with this apicomplexan. Such a study of rodents might well be done in parallel with investigations of other wild mammals such as raccoons and squirrels, which are known to carry Hepatozoon spp. Raccoons may be of especial interest because muscle lesions associated with $H$. procyonis infections in this host are very similar to those seen in dogs with $\mathrm{ACH}$.

\section{Vertical Transmission}

Transplacental transfer of Hepatozoon canis in Japanese dogs has been reported (49). H. griseisciuri, a parasite of the squirrel ( $S$. carolinensis), is reported to be transmitted transplacentally (11). There are no recent studies of H. americanum designed to determine whether transmission to dogs is possible by means other than ingestion of sporulated oocysts. Attempts to transmit what was probably $H$. americanum in the early 1980s by feeding tissues from naturally infected dogs in Texas to susceptible dogs or by injecting similar material were unsuccessful, and the results were inconclusive (51). Young pups have in Texas been found to have naturally occurring hepatozoonosis (51). The observations of H. canis in dogs in Japan and of naturally infected young pups in Texas suggest that transplacental transfer of $H$. americanum might occur in dogs, but this phenomenon has not been demonstrated experimentally. We have seen pups as young as 11 weeks old with naturally occurring $\mathrm{ACH}$ (unpublished data).

\section{Confirmation of the Serologic Approach to Diagnosis}

Studies are in progress to validate further the value of the enzyme-linked immunosorbent assay that was developed recently (44). It appears to be both sensitive and specific and could be used as an aid to determine the extent to which the range of $\mathrm{ACH}$ is expanding. With adaptations to screen sera from rodents and other potential wild vertebrate hosts, it might be very helpful in recognizing which hosts to focus on in an effort to clarify the endemic cycle of $H$. americanum.

\section{Confirmation of the Therapeutic Approach}

Observations by workers at Auburn University suggest that the therapeutic protocol developed there is effective in markedly reducing the severity of $\mathrm{ACH}$ in naturally infected dogs. The regimen appears not to be effective in clearing dogs of infection (40). Our observation that carefully nursed dogs can self-cure (24) of disease manifestations with nothing more than aspirin for pain control while remaining infectious for ticks suggests that more work is needed to address therapy. It must be said, however, that infected dogs probably represent a minimal risk to other dogs because immature $A$. maculatum ticks do not favor dogs as hosts. Thus, Gulf Coast ticks probably are rarely infected under natural conditions by feeding on dogs. Moreover, not only do infected dogs probably not represent a threat to other dogs, but also they are unlikely to contribute effectively to maintenance of the endemic cycle of $H$. americanum. In other words, dogs are probably more or less accidentally inserted into an endemic cycle that would continue without their involvement. Unfortunately for dogs and their owners, this chance exposure commonly results in severe disease and sometimes in death. 


\section{Animal Models of Disease}

Two phenomena that are consistently observed in dogs with $\mathrm{ACH}$ may be worthy of study for reasons related to the health of humans and other animals and to general biological principles. Disseminated periosteal bone proliferation has been found consistently in canids $(32,56)$ experimentally infected with $H$. americanum. Discovery of inciting factors could prove useful in studies of hypertrophic osteoarthropathy in humans. Likewise, determining the agent responsible for the highly vascular nature of granulomas that are consistently seen in dogs (55) with $\mathrm{ACH}$ might prove useful in studies of angiogenesis.

\section{ACKNOWLEDGMENTS}

This study was supported in part by the Wendell H. \& Nellie G. Krull Professorship in Veterinary Parasitology at Oklahoma State University College of Veterinary Medicine.

We thank Margaret Ewing for editorial suggestions and Diana Moffeit and Betty Handlin for manuscript preparation.

\section{REFERENCES}

1. Baneth, G., A. Harmelin, and B.-Z. Presentey. 1995. Hepatozoon canis infection in two dogs. J. Am. Vet. Med. Assoc. 206:1891-1894.

2. Baneth, G., and B. Weigler. 1997. Retrospective case-control study of hepatozoonosis in dogs in Israel. J. Vet. Intern. Med. 11:365-370.

3. Baneth, G., J. R. Barta, V. Shkap, D.S. Martin, D. K. Macintire and N. Vincent-Johnson. 2000. Genetic and antigenic evidence supports the separation of Hepatozoon canis and Hepatozoon americanum at the species level. J. Clin. Microbiol. 38:1298-1301.

4. Baneth, G., J. S. Mathew, V. Shkap, D. K. Macintire, J. R. Barta, and S. A. Ewing. 2003. Canine hepatozoonosis: two disease syndromes caused by separate Hepatozoon spp. Trends Parasitol. 19:27-31.

5. Barker, R. W., A. A. Kocan, S. A. Ewing, and R. P. Wettemann. Seasonal occurrence of the Gulf Coast tick (Acari: Ixodidae) on wild and domestic mammals in north-central Oklahoma. J. Med. Entomol., in press.

6. Barta, J. R. 2000. Suborder Adeleorina Leger, 1911, p. 308-318. In J. J. Lee (ed.), Illustrated guide to the protozoa, 2nd ed., vol. 1. Allen Press, Lawrence, Kans.

7. Barta, J. R. 2001. Molecular approaches for inferring evolutionary relationships among protistan parasites. Vet. Parasitol. 101:175-186.

8. Barton, C. L., E. A. Russo, T. M. Craig, and R. W. Green. 1985. Canine hepatozoonosis: a retrospective study of 15 naturally occurring cases. J. Am. Anim. Hosp. Assoc. 21:125-134.

9. Bentley, C. A. 1905. Preliminary note upon a leucocytozoon of the dog. Br. Med. J. 1:988.

10. Christophers, S. R. 1907. The sexual cycle of Leukocytozoon canis in the tick. Sci. Mem. Off. Med. Sanit. Dept. Gov. India New Ser. 28:1-11.

11. Clark, G. M. 1958. Hepatozoon griseisciuri n. sp.; a new species of Hepatozoon from the grey squirrel (Sciurus carolinensis Gmelin, 1788), with studies on the life cycle. J. Parasitol. 44:52-63.

12. Clark, K. A., R. M. Robinson, L. L. Weishuhn, T. J. Galvin, and K. Howath. 1973. Hepatozoon procyonis infections in Texas. J. Wildl. Dis. 9:182-193.

13. Craig, T. M., J. E. Smallwood, K. W. Knauer, and J. P. McGrath. 1978 Hepatozoon canis infection in dogs: clinical, radiographic and hematological findings. J. Am. Vet. Med. Assoc. 173:967-972.

14. Craig, T. M., L. P. Jones, and R. M. Nordgren. 1984. Diagnosis of Hepatozoon canis by muscle biopsy. J. Am. Anim. Hosp. Assoc. 20:301-303

15. Craig, T. M. 1998. Hepatozoonosis, p. 458-465. In C. E. Greene (ed.), Infectious diseases of the dog and cat, 2nd ed. The W. B. Saunders Co., Philadelphia, $\mathrm{Pa}$

16. Cummings, C. A. 2001. Ph.D. thesis. A morphologic and immunologic study of American canine hepatozoonosis. Oklahoma State University, Stillwater.

17. Davis, D. S., R. M. Robinson, and T. M. Craig. 1978. Naturally occurring hepatozoonosis in a coyote. J. Wildl. Dis. 14:244-246.

18. Desser, S. S. 1990. Tissue "cysts" of Hepatozoon griseisciuri in the grey squirrel, Sciurus carolinensis: the significance of these cysts in species of Hepatozoon. J. Parasitol. 76:257-259.

19. Droleskey, R. E., S. H. Mercer, J. R. DeLoach, and T. M. Craig. 1993. Ultrastructure of Hepatozoon canis in the dog. Vet. Parasitol. 50:83-99.

20. Drost, W. T., C. A. Cummings, J. S. Mathew, R. J. Panciera, and J. C. H. Ko. 2003. Determination of time of onset and location of early skeletal lesions in young dogs experimentally infected with Hepatozoon americanum using bone scintigraphy. Vet. Radiol. Ultrasound 44:86-91.

21. Ewing, S. A., R. J. Panciera, J. S. Mathew, C. A. Cummings, and A. A. Kocan. 2000. American canine hepatozoonosis: an emerging disease in the New World. Ann. N. Y. Acad. Sci. 916:81-92.
22. Ewing, S. A., J. G. DuBois, J. S. Mathew, and R. J. Panciern. 2002. Larval Gulf Coast ticks (Amblyomma maculatum) [Acari: Ixodidae] as host for Hepatozoon americanum [Apicomplexa: Adeleorina]. Vet. Parasitol. 103:4351.

23. Ewing, S. A., J. S. Mathew, and R. J. Panciera. 2002. Transmission of Hepatozoon americanum (Apicomplexa: Adeleorina) by Ixodids (Acari: Ixodidae). J. Med. Entomol. 39:631-634.

24. Ewing, S. A., R. J. Panciera, and J. S. Mathew. 2003. Persistence of Hepatozoon americanum [Apicomplexa: Adeleorina] in a naturally infected $\operatorname{dog}$. J. Parasitol. 89:611-613.

25. Furman, D. P. 1966. Hepatozoon balfouri (Laveran, 1905): sporogonic cycle, pathogenesis, and transmission by mites to jerboa hosts. J. Parasitol. 52:373382

26. Gaunt, P. S., S. D. Gaunt, and T. M. Craig. 1983. Extreme neutrophilic leukocytosis in a dog with hepatozoonosis. J. Amer. Vet. Med. Assoc. 192: 409-410.

27. Gosset, K. A., S. D. Gaunt, and D. S. Aja. 1985. Hepatozoonosis and ehrlichiosis in a dog. J. Am. Anim. Hosp. Assoc. 21:265-267.

28. Harmelin, A., J. P. Dubey, B. Yakobson, A. Nyska, and U. Orgad. 1992. Concurrent Hepatozoon canis and Toxoplasma gondii infections in a dog. Vet. Parasitol. 43:131-136.

29. Inokuma, H., M. Okuda, K. Ohno, K. Shimoda, and T. Onishi. 2002. Analysis of the 18S rRNA gene sequence of a Hepatozoon detected in two Japanese dogs. Vet. Parasitol. 106:265-271.

30. James, S. P. 1905. On a parasite found in the white corpuscles of the blood of dogs. Sci. Mem. Off. Med. Sanit. Dept. Govt. India New Ser. 14:1-12.

31. Kocan, A. A., M. Breshears, R. J. Panciera, S. A. Ewing, and R. W. Barker. 1999. Naturally occurring hepatozoonosis in coyotes from Oklahoma. J. Wildl. Dis. 35:86-89.

32. Kocan, A. A., C. A. Cummings, R. J. Panciera, J. S. Mathew, S. A. Ewing, and R. W. Barker. 2000. Naturally occurring and experimentally transmitted Hepatozoon americanum in coyotes from Oklahoma. J. Wildl. Dis. 36:149143.

33. Krotoski, W. A. 1989. The hypnozoite and malarial relapse. Prog. Clin Parasitol. 1:1-19.

34. Krull, W. H. 1969. Notes in veterinary parasitology. University Press of Kansas, Lawrence.

35. Laird, M. 1959. Malayan protozoa 2. Hepatozoon Miller (Sporozoa: Coccidia), with an unusual record for $H$. canis (James). J. Protozool. 6:316-319.

36. Landau, I. 1973. A comparison of the life cycles of Toxoplasma and Hepatozoon, with reference to the general phenomenon and the role of cyst formation in the coccidian. Ann. Trop. Med. Parasitol. 67:403-407.

37. Lindsay, D. S., J. M. Butler, and B. L. Blagburn. 1997. Efficacy of decoquinate against Neospora caninum tachyzoites in cell cultures. Vet. Parasitol. 68:35-40.

38. Lysenko, A. Y., A. E. Beljaev, and V. M. Rybalka. 1977. Population studies of Plasmodium vivax. I. The theory of polymorphism of sporozoites and epidemiological phenomena of tertian malaria. Bull. W. H. O. 55:541-549.

39. MacIntire, D. K., N. Vincent-Johnson, A. R. Dillon, B. Blagburn, D. L. Lindsay, E. M. Whitley, and C. Banfield. 1997. Hepatozoonosis in dogs: 22 cases (1989-1994). J. Am. Vet. Med. Assoc. 210:916-922.

40. MacIntire, D. K., N. A. Vincent-Johnson, C. W. Kane, D. S. Lindsay, B. L. Blagburn, and A. R. Dillon. 2001. Treatment of dogs infected with Hepatozoon americanum: 53 cases (1989-1998). J. Am. Vet. Med. Assoc. 218:77-82.

41. Mathew, J. S., S. A. Ewing, R. J. Panciera, and J. P. Woods. 1998. Experimental transmission of Hepatozoon americanum Vincent-Johnson et al., 1997 to dogs by the Gulf Coast tick, Amblyomma maculatum Koch. Vet. Parasitol. 80:1-14.

42. Mathew, J. S., S. A. Ewing, R. J. Panciera, and K. M. Kocan. 1999. Sporogonic development of Hepatozoon americanum (Apicomplexa) in its definitive host, Amblyomma maculatum (Acarina). J. Parasitol. 85:1023-1031.

43. Mathew, J. S., R. A. Van Den Bussche, S. A. Ewing, J. R. Malayer, B. R. Latha, and R. J. Panciera. 2000. Phylogenetic relationships of Hepatozoon (Apicomplexa: Adeleorina) based on molecular, morphologic, and life cycle characters. J. Parasitol. 86:366-372.

44. Mathew, J. S., J. T. Saliki, S. A. Ewing, T. W. Lehenbauer, R. J. Panciera, J. R. Malayer, C. A. Cummings, and A. A. Kocan. 2001. An indirect enzymelinked immunosorbent assay for diagnosis of American canine hepatozoonosis. J. Vet. Diagn. Investig. 13:17-21.

45. McCully, R. M., P. A. Basson, R. D. Bigalke, V. DeVos, and E. Young. 1975. Observations on naturally acquired hepatozoonosis of wild carnivores and dogs in the Republic of South Africa. Onderstepoort J. Vet. Sci. 42:117-134.

46. Mercer, S. H. and T. M. Craig. 1988. Comparison of various staining procedures in the identification of Hepatozoon canis gamonts. Vet. Clin. Pathol. 17:63-65.

47. Mercer, S. H., L. P. Jones, J. H. Rappole, D. Twedt, L. L. Laack, and T. M. Craig. 1988. Hepatozoon sp. in wild carnivores in Texas. J. Wildl. Dis. 24: 574-576.

48. Murata, T., K. Shiramizu, Y. Hara, M. Inoue, K. Shimoda, and S. Nakama 1991. First case of Hepatozoon canis infection of a dog in Japan. J. Vet. Med. Sci. 53:1097-1099. 
49. Murata, T., M. Inoue, S. Tateyama, Y. Taura, and S. Nakama. 1993. Vertica transmission of Hepatozoon canis in dogs. J. Vet. Med. Sci. 55:867-868.

50. Murata, T., M. Inoue, Y. Taura, S. Nakama, H. Abe, and K. Fujisaki. 1995. Detection of Hepatozoon canis oocysts from ticks collected from the infected dogs. J. Vet. Med. Sci. 57:111-112.

51. Nordgren, R. M., and T. M. Craig. 1984. Experimental transmission of the Texas strain of Hepatozoon canis. Vet. Parasitol. 16:207-214.

52. O'Dwyer, L. H., C. L. Massard, and J. C. P. de Souza. 2001. Hepatozoon canis infection associated with dog ticks of rural areas of Rio de Janeiro State, Brazil. Vet. Parasitol. 94:143-150.

53. Panciera, R. J., N. T. Gatto, M. A. Crystal, R. G. Helman, and R. W. Ely 1997. Canine hepatozoonosis in Oklahoma. J. Am. Anim. Hosp. Assoc. 33: 221-225.

54. Panciera, R. J., S. A. Ewing, C. A. Cummings, A. A. Kocan, M. A. Breshears, and J. C. Fox. 1998. Observations on tissue stages of Hepatozoon americanum in 19 naturally infected dogs. Vet. Parasitol. 78:265-276.

55. Panciera, R. J., S. A. Ewing, J. S. Mathew, T. W. Lehenbauer, C. A. Cummings, and J. P. Woods. 1999. Canine hepatozoonosis: comparison of lesions and parasites in skeletal muscle of dogs experimentally or naturally infected with Hepatozoon americanum. Vet. Parasitol. 82:261-272.

56. Panciera, R. J., J. S. Mathew, S. A. Ewing, C. A. Cummmings, W. T. Drost, and A. A. Kocan. 2000. Skeletal lesions of canine hepatozoonosis caused by Hepatozoon americanum. Vet. Pathol. 37:225-230.

57. Panciera, R. J., J. S. Mathew, C. A. Cummings, J. C. Duffy, S. A. Ewing, and A. A. Kocan. 2001. Comparison of tissue stages of Hepatozoon americanum in the dog using immunohistochemical and routine histologic methods. Vet. Pathol. 38:422-426.

58. Semtner, P. J., and J. A. Hair. 1973. Distribution, seasonal abundance, and hosts of the Gulf Coast tick in Oklahoma. Ann. Entomol. Soc. Am. 66:12641268.

59. Smith, T. G. 1996 The genus Hepatozoon (Apicomplexa: Adeleina). J. Parasitol. 82:565-585.

60. Strickland, R. K., R. R. Gerrish, J. L. Hourrigan, and G. O. Schubert. 1976 Ticks of veterinary importance. Animal and Plant Health Inspection Service handbook 485. U.S. Department of Agriculture. Government Printing Office, Washington, D.C

61. Van Heerden, J., J. G. L. Mills, J. J. Van Vuuren, P. J. Kelly, and M. J. Dreyer. 1995. An investigation into the health status and diseases of wild dogs (Lyacaon pictus) in the Kruger National Park. J. S. Afr. Vet. Assoc. 66:18-27.

62. Vincent-Johnson, N. A., D. K. MacIntire, D. S. Lindsay, S. D. Lenz, G. Baneth, V. Shkap, and B. L. Blagburn. 1997. A new Hepatozoon species from dogs: description of the causative agent of canine hepatozoonosis in North America. J. Parasitol. 83:1165-1172.

63. Vincent-Johnson, N. A., D. K. MacIntire, and G. Baneth. 1997. Canine hepatozoonosis: pathophysiology, diagnosis and treatment. Compend. Cont. Ed. Pract. Vet. 19:51-65.

64. Wenyon, C. M. 1926. Protozoology: a manual for medical men, veterinarians and zoologists, vol. II, p. 1085. Baillere, Tindall and Cox Ltd., London, United Kingdom. 